\title{
Direct Interaction of Deoxyribonucleic Acid with the Quantum Vacuum that May Lead to Cumulative Damages with Time
}

Meis C*
National Institute for Nuclear Science and Technology, CEA - Centre d'Etudes de Saclay, France

*Corresponding author: Constantin Meis, National Institute for Nuclear Science and Technology, CEA, Centre d'Etudes de Saclay, 91191 Gif-sur-Yvette, France, Email: constantin.

\section{Research Article}

Volume 5 Issue 1

Received Date: January 13, 2021

Published Date: February 02, 2021 meis@cea.fr

\section{Abstract}

The aim of this study is to make a detailed and comparative topological analysis of the single photons and the Deoxyribonucleic Acid (DNA) geometrical structures in order to deduce possible interaction mechanisms, particularly with the quantum vacuum fluctuations. As revealed by the experimental evidence and advanced quantum electrodynamics calculations the quantum vacuum is a sea of transient photons of all wavelengths whose fluctuations interact with matter. Single photon topology and structure is quite characteristic and can be represented by a three dimensional helicoid configuration of the vector potential amplitude over a wavelength. The geometrical configurations of the A DNA, B DNA and Z DNA macromolecules are also known precisely and appear to be similar to that of the photon. Using experimental data and recent quantum electrodynamics theoretical results we put in evidence for the first time that the right-hand canonical B DNA has exactly an identical geometrical configuration with right-hand circular polarization photons having a wavelength equal to the B DNA pitch per turn of helix. Conversely, we show that the radial amplitudes of the right-hand A DNA and the left-hand Z DNA are slightly bigger and smaller respectively compared to photon trains having wavelengths equivalent to the corresponding pitch per helix distances. The amplitudes of the photons electric and magnetic fields are also calculated and revealed to be considerably high locally. We conclude that the DNA alterations by homothetic photons in the soft X-rays range, especially those due to the quantum vacuum fluctuations, may be quite significant contributing to the DNA damage accumulation with time and are worthy to be extensively studied.

Keywords: DNA topology; Single photon structure; Electromagnetic vacuum fluctuations; Photons; DNA homothetic interaction

\section{Introduction}

A synthesis of the experimental results, as well as advanced quantum electrodynamics calculations, have shown that single photons are wave-corpuscles extended over a wavelength along the propagation axis [1-6], having a local radial extension of the order of a quarter of the wavelength [7-9] and guided by a non-local vector potential wave function. The photon quantized vector potential amplitude moves helicoidally over a period with circular (right or left) polarization perpendicular to the propagation axis [4-6,10-
12]. In addition, it has been shown that the electromagnetic vacuum, a cosmic field permeating all of space, is composed of fluctuating transient photons of all modes and polarizations [13-15]. Obviously, the helicoid configuration of the electromagnetic field is analogue to that of the DNA macromolecules. Hence, a direct interaction between the DNA and photons with equivalent wavelengths ranged in the soft X-rays spectrum will inevitably provoke strong structural and chemical modifications with physiological consequences. Furthermore, even in absence of any external radiation, the DNA macromolecules are immersed 
in the electromagnetic vacuum entailing a permanent coupling with transient homothetic vacuum photons with unknown consequences in the DNA physiology and stability. Consequently, it is of high importance to investigate explicitly the topological configurations and the electric and magnetic fields amplitudes of the homothetic photons within the DNA macromolecules. In what follows, we make a detailed and comparative analysis of this particular physical aspect and we evaluate the electric and magnetic fields strengths of the homothetic photons aligned along the nitrogen bases ACGT (adenine, cytosine, guanine, and thymine) that may lead to alterations with time.

\section{Photons and the Electromagnetic Quantum} Vacuum

We recall briefly the single photon geometrical representation. In quantum electrodynamics theory, photons are considered as point particles described by an associated wave [4-6,16-18]. The point particle concept of the photon is an efficient mathematical tool permitting to describe states before and after interaction with the quantized energy levels of atoms and molecules. In reality, the experimental evidence has shown that the photon is not a point particle and that it is impossible to conceive a single $k$-mode photon within a length less than the wavelength [1-5]. Consequently, it is impossible to detect precisely a photon at a given coordinate of the propagation axis while the experiments have shown that the detection probability has a physical sense only within a volume equivalent to the cube of the wavelength $[2,4,5]$. This entails that the photon radial expansion is roughly shorter than the wavelength, which indeed has been confirmed experimentally [8-10]. A synthesis of the experimental facts $[5,10,15]$ yields that a single $k$-mode photon is a quantum of the electromagnetic field, a wavecorpuscle extended over the wavelength $\lambda_{k}$ along the propagation axis. It has a lateral extension $\eta_{k}$ shorter than the wavelength, with the quantized vector potential amplitude $\alpha_{0 k}$ oscillating over a period at the angular frequency $\omega_{k}$ with circular (right or left) polarization. The whole wave-corpuscle packet carries the energy $E_{k}=\hbar \omega_{k}$,

where $\hbar=h / 2 \pi$ is Planck's reduced constant, and has a momentum $\vec{p}_{k}=\hbar \vec{k}$ while it propagates guided by a nonlocal vector potential wave function.

$\vec{\alpha}_{k \lambda}(\vec{r}, t)=\alpha_{0 k}\left(\hat{\varepsilon}_{k \lambda} e^{i\left(\vec{k} \cdot \vec{r}-\omega_{k} t+\theta\right)}+\hat{\varepsilon}_{k \lambda}^{*} e^{-i\left(\vec{k} \cdot \vec{r}-\omega_{k} t+\theta\right)}\right)$

where $|\vec{k}|=\omega_{k} / c$ is the wave-vector, $c$ the speed of light in vacuum and $\lambda$ takes only two values corresponding to left or right circular polarization expressed by the complex unit vector $\hat{\varepsilon}_{k \lambda}$.

The vector potential amplitude $\alpha_{0 k}$ for a single free of cavity $k$-mode photon with angular frequency $\omega_{k}$ writes $[6,15,19-21]$.

$$
\alpha_{0 k}=\xi \omega_{k} \text { with }|\xi|=\left|\frac{\hbar}{4 \pi e c}\right|=1.74710^{-25} \text { Volt m}^{-1} s^{2}
$$

where $e$ is the electron-positron charge.

Hence, the vector potential of a free single photon in the plane wave representation writes

$$
\vec{\alpha}_{k \lambda}(\vec{r}, t)=\xi \omega_{k}\left(\hat{\varepsilon}_{k \lambda} e^{i\left(\vec{k} \cdot \vec{r}-\omega_{k} t+\theta\right)}+\hat{\varepsilon}_{k \lambda}^{*} e^{-i\left(\vec{k} \cdot \vec{r}-\omega_{k} t+\theta\right)}\right)=\omega_{k} \vec{\Xi}_{k \lambda}(\vec{r}, t)
$$

Various experiments have demonstrated that the radial spatial extension of a $k$-mode photon is approximately $\eta_{k} \approx 0.25 \lambda_{k}$ [7-9] while a theoretical analysis [6,15,22]

yields the value

$$
\eta_{k}=\sqrt{8 \alpha} \lambda_{k} \approx 0.242 \lambda_{k}
$$

with $a=1 / 137$ the fine structure constant.

Considering a circular polarization the summation of the electromagnetic field energy density over a wavelength yields the energy of a single photon $\hbar \omega_{k}[6,15]$. Thus, the photon vector potential follows a precession with circular polarization over a wavelength $\lambda_{k}$ and extends laterally over a radius of the order $\eta_{k}$ describing a periodic helicoid curve along the propagation axis. It generates locally an electric and magnetic field, $\vec{\varepsilon}_{k}$ and $\vec{\beta}_{k}$ respectively, which are perpendicular to each other $\left(\vec{\varepsilon}_{k} \perp \vec{\beta}_{k}\right)$ and orthogonal to the propagation axis $\left(\vec{\varepsilon}_{k} \cdot \vec{k}=\vec{\beta}_{k} \cdot \vec{k}=0\right)$. Their amplitudes are proportional to the square of the angular frequency $[6,12,15]$.

$$
\begin{gathered}
\left|\vec{\varepsilon}_{k}\right|=\left|-\partial \vec{\alpha}_{k \lambda}(\vec{r}, t) / \partial t\right|=\xi \omega_{k}^{2} ; \\
\left|\vec{\beta}_{k}\right|=\left|\vec{\nabla} \times \vec{\alpha}_{k \lambda}(\vec{r}, t)\right|=\xi \omega_{k}^{2} / c
\end{gathered}
$$

The zero-frequency level corresponds to zero energy electromagnetic field. At that level, the vector potential is also zero but the fundamental field $\vec{\Xi}_{k \lambda}$ in equation (3) does not vanish and reduces to 


$$
\vec{\Xi}_{0 \lambda}=\xi\left(\hat{\varepsilon}_{\lambda} e^{i \phi}+\hat{\varepsilon}_{\lambda}^{*} e^{-i \phi}\right)
$$

$\vec{\Xi}_{0 \lambda}$ is the Electromagnetic Field Ground State, a real field permeating all of space $\left(\omega_{k} \rightarrow 0 \Rightarrow \lambda_{k} \rightarrow \infty\right)$ having zero-

energy, zero vector potential as well as zero electric and magnetic fields (dark light). Under this condition, the field $\vec{\Xi}_{0 \lambda}$ is a real vacuum component, the electromagnetic quantum vacuum, having electric potential dimension. Obviously, photons are oscillations of the electromagnetic quantum vacuum [6,13-15,20,21]. Furthermore, the electromagnetic field ground state may have a complex structure yet to be discovered $[5,10,15,23]$.

Heisenberg's energy-time uncertainty relation applied to equation (5) entails directly that the vector potential amplitude is also subject to a fluctuation uncertainty

$$
\delta E_{k} \cdot \delta t \approx \hbar \quad=>\delta \alpha_{0 k} \cdot \delta t \approx \xi(7)
$$

Consequently, fluctuations of the quantum electromagnetic vacuum may generate transient states of photons of any wavelength at any point in space.

\section{Topological Analogy between DNA and Photons. DNA Interaction with Homothetic Photons}

The DNA structure is well known and widely commented in the literature [24-26]. This nucleic acid is composed of nucleotides molecules each containing a phosphate group, a 5-carbon sugar called deoxyribose and a nitrogen base. The molecules are distributed over a double helix geometrical configuration with the nitrogen bases ACGT aligned along the helix diameter.
We denote by $\lambda_{D N A}$, the periodic distance of the helix strands corresponding to "DNA wavelength", and by $\delta_{D N A}$ the thickness of the strands borders composed of deoxyribose and phosphate groups. The diameter of the helix is $\rho_{D N A}$ while $\eta_{D N A}$ is the mean radius corresponding to the distance from the helix axis to the middle of the border molecules, that is

$$
\eta_{D N A}=\left(\rho_{D N A}-\delta_{D N A}\right) / 2
$$

The measured values for the geometric characteristics of both right-hand A and B DNA $[27,28]$ as well as of the left-hand Z DNA [29,30] are given in Table 1 and they are compared to those of photons with equivalent wavelengths. The corresponding photon frequencies $v_{k}=\omega_{k} / 2 \pi$, laying in the range extreme-UV to soft-X rays, as well as the photon electric and magnetic field amplitudes (rounded to the nearest integer) are also given.

It appears that the topological structure of the DNA is similar to a train of photons with equal wavelengths $\lambda_{k}=\lambda_{D N A}$ and identical polarization, right-hand for the A

DNA and B DNA and left-hand for the Z DNA. It is worth noting (in bold on Table 1) the surprising coincidence for the canonical B DNA which has exactly the same geometrical

configuration and radial extension $\left(\eta_{B D N A}=\eta_{k}\right)$ with a righthand polarization photon having the same wavelength $\left(\lambda_{B D N A}=\lambda_{k}\right)$ entailing a perfect homothetic physical

structure. For the left-hand Z DNA a symmetrical left-hand photon train with same wavelength covers totally the macromolecule. Conversely, the A DNA has a lateral extension significantly larger than that of an equal wavelength photon.

\begin{tabular}{|c|c|c|c|c|c|c|c|c|c|}
\hline & $\lambda_{D N A}$ & $\eta_{D N A}$ & $\rho_{D N A}$ & $\delta_{D N A}$ & $\lambda_{k}$ & $\eta_{k}$ & $v_{k}(\mathrm{PHz})$ & $\varepsilon_{k}\left(\mathrm{~V} \mathrm{~nm}^{-1}\right)$ & $\beta_{k}(T)$ \\
\hline A DNA & 2.82 & 1.17 & 2.6 & 0.256 & 2.82 & 0.68 & 106.3 & 78 & 260 \\
\hline B DNA & $\mathbf{3 . 3 8}$ & $\mathbf{0 . 8 3}$ & 2.0 & 0.338 & $\mathbf{3 . 3 8}$ & $\mathbf{0 . 8 2}$ & 88.7 & 54 & 181 \\
\hline Z DNA & 4.56 & 0.71 & 1.8 & 0.380 & 4.56 & 1.10 & 65.7 & 30 & 99 \\
\hline
\end{tabular}

${ }^{1}$ All length values are in $\mathrm{nm}$

Table 1: DNA geometric values compared to k-mode photons with equivalent wavelengths.

The homothetic photons electric and magnetic fields are aligned on the helix axis along the nitrogen bases ACGT and can directly induce various effects, particularly for the B DNA, such as bonds break up, dissociation-recombination process, chemical reactions, ionizations,...etc. Even if the coupling occurs during short time intervals the high amplitudes of the electric and magnetic field strengths, given in Table 1 , could be sufficient to produce permanent structural and chemical modifications with physiological consequences.

In fact, vacuum fluctuations occur permanently and everywhere in space at all wavelengths and in all directions. 
DNA macromolecules have also a random distribution in space in all directions. Consequently, even in absence of real photons the DNA is immersed permanently in the electromagnetic quantum vacuum and due to its photonlike geometry it may interact directly with fluctuating transient vacuum photons. The vacuum transient photons have naturally intrinsic circular polarization, they do not have time to propagate and act locally as electric and magnetic perturbations. Hence, over long time intervals, the superposed effects of successive random events entail that the possibility for direct alteration of the B-DNA due to vacuum fluctuations photons with similar wavelength might not be negligible.

\section{Conclusions}

We have shown that the topological configurations of the $\mathrm{A}, \mathrm{B}$ and $\mathrm{Z}$ DNA are similar to photons with equivalent wavelengths and polarizations. There is an intriguing perfect homothetic coincidence with exactly the same lateral extension between the canonical B DNA and a circular righthand polarized photon with wavelength $\lambda_{k}=3.38 \mathrm{~nm}$. Hence, there is a direct coupling between homothetic photons and the B DNA macromolecules. The photons electric and magnetic fields, whose values are extremely high locally, are aligned exactly along the nitrogen bases ACGT on the helix axis and may lead to considerable alterations. Hence, transient photons from the quantum vacuum fluctuations having identical wavelengths and polarizations with the DNA macromolecules may act locally upon the ACGT bases as permanent random electromagnetic perturbations. Consequently, these effects are worthy to be investigated extensively because they may have direct consequences by inducing damage accumulation with time influencing the DNA evolution.

\section{References}

1. Akhiezer AI, Berestetskii BV (1965) Quantum Electrodynamics. Interscience Publishers, New York, USA.

2. Mandel L (1966) Configuration-Space Photon Number Operators in Quantum Optics. Phys Rev 144(4): 1071.

3. Pfleegor RL, Mandel L (1967) Interference of Independent Photon Beams. Phys Rev 159(5): 1084.

4. Grynberg G, Aspect A, Fabre C (2010) Introduction to Quantum Optics. Cambridge University Press, New York, USA, pp: 697.

5. Garrison JC, Chiao RY (2008) Quantum Optics. Oxford University Press, New York, USA.
6. Meis C (2017) Light and Vacuum. $2^{\text {nd }}($ Edn.), World Scientific, Singapore.

7. Robinson HL (1953) Diffraction Patterns in Circular Apertures Less Than One Wavelength in Diameter. J Appl Phys 24(1): 35.

8. Hadlock RK (1958) Diffraction Patterns at the Plane of a Slit in a Reflecting Screen. J Appl Phys 29: 918.

9. Hunter G, Wadlinger RLP (1989) Photons and Neutrinos as Electromagnetic Solitons. Physics Essays 2(2): 158172.

10. Jeffers S, Roy S, Vigier JP, Hunter G (1997) The present status of the quantum theory of light. $1^{\text {st }}$ (Edn.), Kluwer Academic Publishers, Boston, USA, pp: 554.

11. Smith BJ, Raymer MG (2007) Photon wave functions, wave-packet quantization of light, and coherence theory. New J Phys 9: 414.

12. Meis C, Dahoo PR (2017) Vector potential quantization and the photon intrinsic electromagnetic properties: Towards nondestructive photon detection. Int Jour Quantum Inf 15(8): 1740003.

13. Meis C (2018) The Electromagnetic field Ground State and the Cosmological Evolution. Jour of Physics Conf Series 1141: 012072.

14. Meis C (2020) Primary role of the quantum electromagnetic vacuum in gravitation and cosmology. IntechOpen.

15. Meis C (2019) Quantized field of single photons. IntechOpen.

16. Ryder LH (1987) Quantum field theory. Cambridge University Press, Cambridge.

17. Milonni PW (2013) The quantum vacuum. $1^{\text {st }}$ (Edn.), Academic Press Inc., London, pp: 522.

18. Weissbluth M (2012) Photon-Atom Interactions. $1^{\text {st }}$ (Edn.), Academic Press, Inc., London.

19. Meis C (2014) Vector potential quantization and the quantum vacuum. Phys Res Int 5.

20. Meis C, Dahoo PR (2016) Vector potential quantization and the photon wave-particle representation. Jour Phys Conf Series 738: 012099.

21. Meis C, Dahoo PR (2017) Quantized vector potential and the photon wave function. Jour of Phys Conf Series 936: 012004 . 
Physical Science \& Biophysics Journal

22. Meis C (1999) Zero-point radiation field. Phys Essays 21(1): 174-181.

23. Meis C, Dahoo PR (2018) The Single Photon State, the Quantum Vacuum and the Elementary Electron/Positron Charge. Am Inst of Physics Conf Proc 2040(1): 020011.

24. Watson JD, Crick FH (1953) Molecular structure of nucleic acids; a structure for deoxyribose nucleic acid. Nature 171: 737-738.

25. Calladine CR, Drew HR, Luise BF, Travers AA (2004) Understanding DNA. The molecule $\&$ how it works. $3^{\text {rd }}$ (Edn.), Academic Press, pp: 352.

26. Saenger W (1984) Principles of nucleic acid structure. $1^{\text {st }}$
(Edn.), Springer-Verlag, New York, USA, pp: 556.

27. Ricardo Arias-Gonzalez J (2014) Single-molecule portrait of DNA and RNA double helices. Integrative Biology 6(10): 904-925.

28. Richard R Sinden (2012) DNA structure and function. $1^{\text {st }}$ (Edn.), Academic Press, London, pp: 398.

29. Rich A, Norheim A, Wang AHJ (1984) The chemistry and biology of left-handed Z-DNA. Annual Review of Biochemistry 53:[791-846.

30. Ho PS (1994) The non-B-DNA structure of d(CA/TG)n does not differ from that of Z-DNA. Proc Natl Acad Sci USA 91(20):"9549-9553. 